\title{
Crescimento e competitividade de biótipos de capim-colchão resistente e suscetível aos herbicidas inibidores da acetil coenzima A carboxilase
}

\author{
Ramiro Fernando López Ovejero(1), Maria do Carmo de Salvo Soares Novo(2), \\ Saul Jorge Pinto de Carvalho(1), Marcelo Nicolai ${ }^{(1)}$ e Pedro Jacob Christoffoleti(1)
}

\begin{abstract}
(1)Escola Superior de Agricultura Luiz de Queiroz, Dep. de Produção Vegetal, Av. Pádua Dias, no 11, Caixa Postal 09, CEP 13418-900 Piracicaba, SP. E-mail: rfloveje@esalq.usp.br, sjpcarvalho@yahoo.com.br, marcelon@esalq.usp.br, pjchrist@esalq.usp.br (2)Instituto Agronômico, Centro de Ecofisiologia e Biofísica, Av. Theodureto de Almeida Camargo, no 1.500, Vila Nova, Caixa Postal 28 , CEP 13075-630 Campinas, SP. E-mail: mcdesalvo@hotmail.com
\end{abstract}

Resumo - O objetivo deste trabalho foi comparar o crescimento e a habilidade competitiva de dois biótipos de capim-colchão (Digitaria ciliaris), um resistente (R) e outro suscetível (S) aos herbicidas inibidores da acetil coenzima A carboxilase. O crescimento dos biótipos foi determinado pela coleta da matéria seca das plantas, aos 14, 21, 25, 28, 34, 42, 49, 57, 65, 72, 78, 86, 101, 111 e 118 dias após emergência (DAE). Os dados de massa de matéria seca foram ajustados ao modelo logístico e, também, utilizados para a obtenção da taxa de crescimento absoluto. Para avaliar a habilidade competitiva intra-específica e interespecífica, foram instalados cinco experimentos com o uso do método substitutivo. Compararam-se os biótipos R e S entre si e cada um destes com a cultura da soja, quando semeada no mesmo dia ou sete dias após a semeadura das plantas daninhas. As proporções de plantas entre as espécies ou biótipos utilizados foram: 5:0; 4:1;3:2; 2:3; 1:4 e 0:5. Os biótipos de capim-colchão apresentaram acúmulo de matéria seca, crescimento absoluto e competição interespecífica semelhantes, e a redução da matéria seca da soja foi similar na presença dos biótipos R e S, o que sugere que ambos os biótipos de capim-colchão possuem a mesma adaptabilidade ecológica.

Termos para indexação: Digitaria ciliaris, competição, método substitutivo, soja.

\section{Growth and competitiveness of biotypes of crabgrass resistant and susceptible to acetyl coenzyme A carboxylase inhibiting herbicides}

\begin{abstract}
The objective of this work was to compare the growth and the competitive ability of two crabgrass (Digitaria ciliaris) biotypes, one resistant (R) and other susceptible (S) to the acetyl coenzyme A carboxylase inhibiting herbicides. Biotypes growth was determined by collecting the plant dry mass at 14, 21, 25, 28, 34, 42, 49, $57,65,72,78,86,101,111$, and 118 days after emergence (DAE). Data of dry mass were adjusted to a logistic model, and were also used to calculate the absolute growth rate. Five experiments were installed to evaluate the intraspecific and interspecific competitive ability, using the substitutive method. The biotypes $\mathrm{R}$ and $\mathrm{S}$ were compared between themselves and each one of them with the soybean crop seeded in the same day or seven days after the seeding of the weeds. Plant proportions between species or biotypes used were: 5:0; 4:1; 3:2; 2:3; 1:4, and 0:5. The biotypes presented similar dry mass accumulation, absolute growth rate and interspecific competition, and the reduction of soybean mass was similar in the presence of $\mathrm{R}$ and $\mathrm{S}$ crabgrass biotypes, which suggests that $\mathrm{R}$ and $\mathrm{S}$ crabgrass biotypes have the same ecological adaptability.
\end{abstract}

Index term: Digitaria ciliaris, competition, substitutive series, soybean.

\section{Introdução}

No Brasil, uma das principais plantas daninhas que infestam a cultura da soja (Glycine max (L.) Merril) é o capim-colchão (Digitaria ciliaris (Retz.) Koel.) que, em razão de sua elevada adaptabilidade ecológica, ocorre freqüentemente nos ambientes produtivos dos Estados do Paraná e de São Paulo.
Em geral, as plantas daninhas interferem na produtividade das culturas por competir por água, luz e nutrientes e, particularmente na cultura da soja, a presença dessas plantas pode causar perdas significativas de produtividade (Spadotto et al., 1994; Fleck et al., 2002), de modo que seu controle tornase indispensável.

Biótipos de capim-colchãoforamidentificados comoresistentes aos herbicidas inibidores da acetil coenzima A carboxilase 
(ACCase), no Estado do Paraná (López Ovejero et al., 2005). Neste caso, estratégias de manejo devem ser adotadas, com atuação tanto na prevenção ao surgimento da resistência, como também para o manejo das populações de plantas daninhas resistentes. Contudo, a atuação no manejo da resistência deve estar baseada em conhecimentos sólidos sobre a biologia dos biótipos resistente e suscetível.

Segundo López Ovejero et al. (2004), os princípios básicos para a prevenção e manejo da resistência são: redução da pressão de seleção, por meio do planejamento criterioso da aplicação de herbicidas, com diferentes mecanismos de ação; adoção de técnicas culturais que afetam a dinâmica populacional das sementes de plantas daninhas, no banco de sementes do solo, e retardam o aumento da frequiência de sementes resistentes neste banco; e conhecimento da adaptabilidade ecológica do biótipo resistente, em relação ao suscetível, para compreender seu comportamento na área afetada.

A adaptabilidade ecológica de um biótipo referese ao seu sucesso evolutivo, que combina as características de crescimento, sobrevivência e reprodução (Holt \& Radosevich, 1983); ou seja, tratase da capacidade que um biótipo possui, dentro de uma população de plantas daninhas, em manter ou aumentar sua proporção ao longo do tempo (Christoffoleti et al., 1997). A adaptabilidade ecológica comparativa do biótipo resistente (R) em relação ao suscetível (S), é um fator que pode influenciar de forma significativa a taxa de evolução da resistência, em sua freqüência inicial, ou mesmo na manutenção da proporção de plantas resistentes dentro da população, quando na ausência da pressão de seleção do herbicida (Warwick \& Black, 1994; Jasieniuk et al., 1996).

Para comparar a adaptabilidade ecológica entre biótipos resistentes e suscetíveis, freqüentemente são utilizadas taxas de germinação, crescimento e produção de sementes. Esses parâmetros permitem detectar possíveis diferenças funcionais e estruturais entre biótipos (Holt \& Radosevich, 1983; Christoffoleti, 2001). Além dessa comparação, é importante avaliar a habilidade competitiva dos biótipos (Warwick \& Black, 1994). Os biótipos mais adaptados são normalmente mais competitivos e capazes de aumentar sua proporção ao longo do tempo e, assim, eliminam os indivíduos menos aptos a ocupar determinado nicho ecológico (Christoffoleti et al., 1997).
O objetivo deste trabalho foi comparar o crescimento e a habilidade competitiva intra-específica e interespecífica de dois biótipos de capim-colchão (Digitaria ciliaris), um resistente (R) e outro suscetível (S) aos herbicidas inibidores da ACCase.

\section{Material e Métodos}

Foram utilizados dois biótipos de capim-colchão, um comprovadamente resistente (R4) aos herbicidas inibidores da ACCase (López Ovejero et al., 2005), coletado em Piraí do Sul, PR (24³3'45"S; 4956'15"W); e outro suscetível (S), coletado em área sem histórico de aplicação de inibidores da ACCase, em Ponta Grossa,

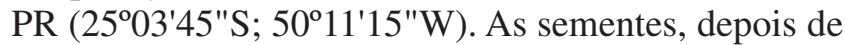
colhidas em fevereiro de 2002, foram acondicionadas em sacos de papel, identificadas (Canto-Dorow, 2001), etiquetadas, multiplicadas e armazenadas em local seco, à temperatura ambiente, até a instalação dos experimentos.

Para análise de crescimento em condições nãocompetitivas, foi conduzido um experimento em casa de vegetação, do Centro de Ecofisiologia e Biofísica (CEB) do Instituto Agronômico (IAC), em Campinas, SP, entre abril e agosto de 2004. Os biótipos R e S de capim-colchão foram semeados diretamente nos vasos, no dia 20 de abril, e em 26 de abril ocorreu a emergência. Aos 14 dias após emergência (DAE), foi realizado o desbaste, tendo-se deixado apenas uma planta por vaso. As parcelas constaram de vasos com capacidade para $5 \mathrm{~L}$, preenchidos com solo argiloso, coletado em área mantida sob pivô central, cuja análise físico-química é apresentada na Tabela 1. Os vasos foram irrigados diariamente.

O delineamento experimental adotado foi o de blocos ao acaso, com 15 tratamentos (avaliações aos 14, 21, $25,28,34,42,49,57,65,72,78,86,101,111$ e 118 DAE) e quatro repetições. As plantas foram coletadas aleatoriamente em cada avaliação e, em seguida, lavadas em água corrente, para a retirada do solo remanescente nas raízes. O material coletado foi secado em estufa de circulação renovada de ar, a $70^{\circ} \mathrm{C}$, até a obtenção de massa constante, quando se mensurou a massa de matéria seca total (MS = parte aérea + raízes), por meio de balança de precisão.

Em cada avaliação, com os valores primários da variável MS, pôde-se calcular a taxa de crescimento absoluto (G, g dia $\left.{ }^{-1}\right)$, a partir da fórmula: $\mathrm{G}=\left(\mathrm{MSt}_{2}-\mathrm{MSt}_{1}\right) /$ $\left(t_{2}-t_{1}\right)$, em que $M_{S t} t_{2}$ e MSt $t_{1}$ são as massas da matéria seca total de duas amostras sucessivas, e $t_{2}$ e $t_{1}$ são os 
dias decorridos entre as duas observações (Calbo et al., 1989; Aguilera et al., 2004).

Os dados de MS e G foram submetidos à análise de variância, com aplicação do teste $\mathrm{F}$, seguido da análise de regressão. Os valores de $\mathrm{G}$ foram ajustados a modelos lineares de quarto grau, enquanto para MS foi adotado o seguinte modelo não-linear logístico: $\mathrm{y}=\mathrm{a}+4 \mathrm{~b}\left\{\exp [-(\mathrm{x}-\mathrm{c}) / \mathrm{d}] /[1+\exp [-(\mathrm{x}-\mathrm{c}) / \mathrm{d}]]^{2}\right\}$, em que y é a variável de interesse, x é o número de dias acumulados e a, b, c e d são parâmetros estimados da equação (b é a amplitude existente entre o ponto máximo e o ponto mínimo, c é o número de dias acumulados, correspondente ao ponto máximo da curva, e a e d são constantes). Este modelo foi o que melhor se adaptou aos dados, com elevado coeficiente de determinação $\left(\mathrm{R}^{2}\right)$ e parâmetros biologicamente aceitáveis.

Para a avaliação da competição intra-específica, foi conduzido um experimento em casa de vegetação, do Dep. de Produção Vegetal, da Escola Superior de Agricultura Luiz de Queiroz (Esalq), em Piracicaba, SP, entre os meses de janeiro e abril de 2005. Utilizou-se o método substitutivo de estudos de interferência entre plantas (Cousens, 1991), tendo-se comparado as associações dos biótipos $\mathrm{R}$ e $\mathrm{S}$ de capim-colchão.

O método substitutivo consiste em manter constante a densidade total da população de plantas e em manter variável a proporção entre as duas espécies ou biótipos (Harper, 1977). As populações das espécies em estudo, chamadas monoculturas, também são incluídas no

Tabela 1. Características físico-químicas do solo utilizado no experimento.

\begin{tabular}{lc}
\hline Variável & Concentração \\
\hline $\mathrm{P}\left(\mathrm{mg} \mathrm{dm}^{-3}\right)$ & 27,0 \\
$\mathrm{MO}\left(\mathrm{g} \mathrm{dm}^{-3}\right)$ & 25,0 \\
$\mathrm{pH} \mathrm{CaCl}$ & 5,5 \\
$\mathrm{~K}\left(\mathrm{mmol}_{\mathrm{c}} \mathrm{dm}^{-3}\right)$ & 4,2 \\
$\mathrm{Ca}\left(\mathrm{mmol}_{\mathrm{c}} \mathrm{dm}^{-3}\right)$ & 29,0 \\
$\mathrm{Mg}\left(\mathrm{mmol}_{\mathrm{c}} \mathrm{dm}^{-3}\right)$ & 15,0 \\
$\mathrm{H}+\mathrm{Al}\left(\mathrm{mmol}_{\mathrm{c}} \mathrm{dm}^{-3}\right)$ & 25,0 \\
$\mathrm{SB}\left(\mathrm{mmol}_{\mathrm{c}} \mathrm{dm}^{-3}\right)$ & 48,2 \\
$\mathrm{CTC}\left(\mathrm{mmol}_{\mathrm{c}} \mathrm{dm}^{-3}\right)$ & 73,2 \\
$\mathrm{~V}(\%)$ & 66,0 \\
$\left.\mathrm{~B}(\mathrm{mg} \mathrm{dm})^{-3}\right)$ & 0,19 \\
$\mathrm{Cu}\left(\mathrm{mg} \mathrm{dm}^{-3}\right)$ & 5,2 \\
$\mathrm{Fe}\left(\mathrm{mg} \mathrm{dm}^{-3}\right)$ & 14,0 \\
$\mathrm{Mn}\left(\mathrm{mg} \mathrm{dm}^{-3}\right)$ & 52,9 \\
$\mathrm{Zn}\left(\mathrm{mg} \mathrm{dm}^{-3}\right)$ & 2,3 \\
Areia total $\left(\mathrm{g} \mathrm{kg}^{-1}\right)$ & 352 \\
Silte $\left(\mathrm{g} \mathrm{kg}^{-1}\right)$ & 133 \\
Argila $\left(\mathrm{g} \mathrm{kg}^{-1}\right)$ & 588 \\
\hline
\end{tabular}

experimento. A premissa básica deste tipo de delineamento é a determinação da produtividade das misturas das duas espécies, em comparação com a produção da monocultura. É importante que este tipo de experimento seja conduzido em uma densidade total, que esteja na faixa da produção final constante, ou seja, a produção de biomassa, por unidade de área, é independente da densidade das plantas naquela área (Radosevich, 1987; Radosevich \& Roush, 1990).

As sementes dos dois biótipos germinaram em caixas de plástico, com capacidade para $2 \mathrm{~L}$, preenchidas com substrato comercial. Quando as plântulas se apresentavam em estádio de uma folha definitiva, foram transplantadas para vasos de plástico, com capacidade para $5 \mathrm{~L}$, preenchidos com o mesmo substrato comercial nas proporções de (R:S): 5:0; 4:1; 3:2; 2:3; 1:4; e 0:5, tendo-se mantido a densidade constante (cinco plantas por vaso). Em termos porcentuais, estas proporções correspondem a 100, 80, 60, 40, 20 e $0 \%$ de plantas do biótipo R e o inverso de plantas do biótipo $\mathrm{S}$, expressam a produção porcentual esperada dos biótipos, se iguais competitivamente.

As plantas foram distribuídas uniformemente nas parcelas. Os vasos foram mantidos em casa de vegetação e irrigados diariamente. $\mathrm{O}$ delineamento experimental adotado foi o de blocos ao acaso, com seis tratamentos e quatro repetições. Por ocasião do estádio fenológico de enchimento das sementes das plantas daninhas, a parte aérea dos biótipos foi coletada separadamente, secada em estufa de circulação renovada de ar, a $70^{\circ} \mathrm{C}$, por dois dias, até massa constante, e foi posteriormente pesada.

Para avaliação da competição interespecífica, foram conduzidos experimentos entre os meses de outubro de 2004 e março de 2005, em casa de vegetação, no CEB do IAC, tendo-se utilizado método substitutivo, para comparar as associações dos biótipos $\mathrm{R}$ ou $\mathrm{S}$ de capim-colchão com plantas de soja. Foram adotadas duas épocas de semeadura das plantas daninhas, em relação às plantas de soja: no mesmo dia (0 dia antes da soja-DAS) e sete dias antes (7 DAS). A segunda época de semeadura (7 DAS) foi utilizada com o objetivo de potencializar a capacidade competitiva dos biótipos de capim-colchão e, desta forma, facilitar a identificação de eventuais diferenças competitivas existentes entre eles.

As proporções utilizadas foram (R ou S:soja): 5:0; 4:1; $3: 2 ; 2: 3 ; 1: 4$ e $0: 5$; tendo-se mantido a densidade constante (cinco plantas por vaso). Em termos porcentuais, estas proporções correspondem a 100, 80, 
60, 40, 20 e 0\% de plantas dos biótipos R ou S, e o inverso de plantas de soja, e expressam a produção porcentual esperada das plantas, se foram iguais competitivamente. As plantas foram distribuídas uniformemente nas parcelas. O delineamento experimental foi o de blocos ao acaso, com seis tratamentos e quatro repetições. Os experimentos foram instalados em vasos com capacidade para $14 \mathrm{~L}$, preenchidos com solo argiloso, coletado em área de pivô central, cuja análise é apresentada na Tabela 1 . Os vasos foram irrigados diariamente. A partir do florescimento das plantas de soja, foram utilizadas adubações com soluções que continham 80 ppm de $\mathrm{P}_{2} \mathrm{O}_{5}$ e de $\mathrm{K}_{2} \mathrm{O}$.

A cultivar de soja utilizada foi IAC-23, proveniente de Pindorama, com $98 \%$ de germinação e pureza. Pouco antes da semeadura, as sementes de soja receberam inoculação de inoculante turfoso comercial (Bradyrhizobium japonicum - SEMIA 5079 (CPAC-15) + SEMIA 587, com $3 \times 10^{9}$ células viáveis por grama de inoculante), na dose de $250 \mathrm{~g}$ por $100 \mathrm{~kg}$ de semente. As sementes dos biótipos $\mathrm{R}$ e $\mathrm{S}$ de capim-colchão foram multiplicadas na casa de vegetação e apresentaram porcentagem de germinação de 67,2 e $77,4 \%$ para o biótipo R e S, respectivamente, avaliadas em testes prévios de germinação.

As semeaduras da cultura da soja e dos biótipos $\mathrm{R}$ e $\mathrm{S}$ de capim-colchão foram realizadas diretamente nos vasos, na profundidade de $3 \mathrm{~cm}$, e a lanço, com cobertura de $1 \mathrm{~cm}$ de solo, respectivamente. Após a emergência, realizou-se o desbaste das plantas, tendo-se deixado as proporções desejadas. Por ocasião do estádio R5.4 da soja (maioria das vagens entre 50 e $75 \%$ de granação), nos quatro experimentos, colheu-se a parte aérea das plantas daninhas e da soja, separadamente por espécie. As amostras foram secadas em estufa a $70^{\circ} \mathrm{C}$, por dois dias, até massa constante e, posteriormente, pesadas.

Os dados dos experimentos de competição intraespecífica e interespecífica foram submetidos à análise convencional para experimentos substitutivos (Radosevich, 1987), que é realizada visualmente, com o auxílio de gráficos com a resposta do rendimento relativo, em função da proporção das espécies. As produções relativas, em todas as proporções, foram calculadas, dividindo-se a produção obtida em diferentes proporções, pela produção média da parcela que continha somente uma espécie de planta (monocultura) (Wit \& Van Den Berg, 1965; Harper, 1977).

\section{Resultados e Discussão}

No experimento de análise de crescimento em condições não competitivas, a análise de variância da MS e do G não apresentou significância para a interação biótipo x época. O efeito simples para biótipos também não foi significativo, o que indica que o acúmulo de massa de matéria seca apresentou comportamento semelhante, para ambos os biótipos estudados. Os valores máximos de MS foram de 5,3 g por planta aos $86 \mathrm{DAE}$, para o biótipo $\mathrm{S}$, e de 4,9 g por planta aos $111 \mathrm{DAE}$, para o biótipo R (Figura 1). Isso pode ser explicado, uma vez que se observou que o estádio de maturação das sementes foi atingido primeiro pelo biótipo $\mathrm{S}$.

A taxa de crescimento absoluto (G) é apresentada na Figura 2. Essa taxa fornece uma estimativa da velocidade média de crescimento das plantas, ao longo do período de observação (Aguilera et al., 2004). Para Vidal \& Trezzi (2000), espécies com elevada $G$ podem levar vantagem competitiva, em virtude da ocupação rápida de um espaço amplo e do

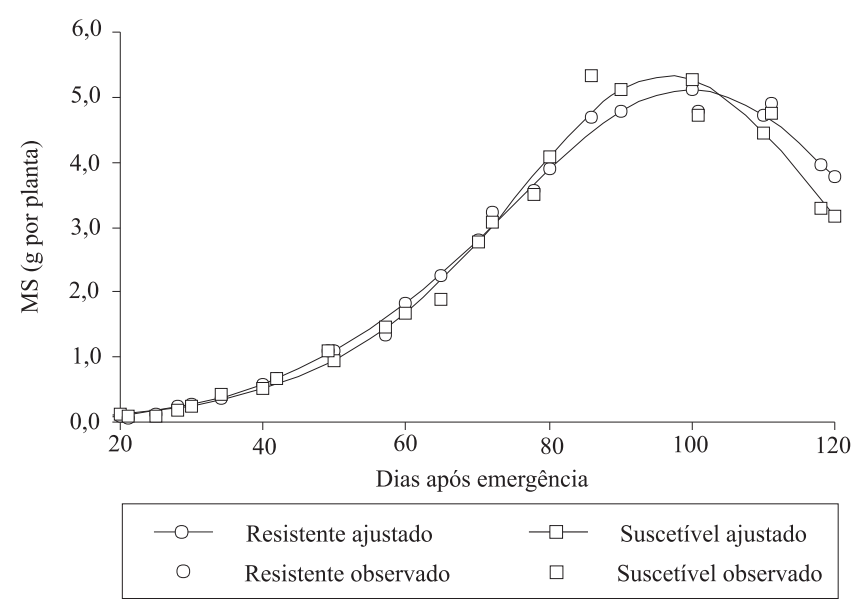

Figura 1. Acúmulo de matéria seca total (MS) apresentado pelos biótipos de capim-colchão, resistente e suscetível aos herbicidas inibidores da ACCase, durante o ciclo de crescimento. A curva representa os valores ajustados da equação não linear, e os pontos equivalem à média dos valores observados. Equações:

$$
\begin{aligned}
& \mathrm{MS}_{\mathrm{RES}}=-0,209+21,36 \frac{\exp -\left[\frac{(\mathrm{x}-99,536)}{18,578}\right]}{\left[1+\exp \left(-\frac{(\mathrm{x}-99,536)}{18,578}\right)\right]^{2}}\left(\mathrm{R}^{2}=0,993\right) ; \\
& \left.\mathrm{MS}_{\mathrm{SUS}}=-0,040+21,54 \frac{\exp -\left[\frac{(\mathrm{x}-96,517)}{15,579}\right]}{\left[1+\exp \left[-\frac{(\mathrm{x}-96,517)}{15,579}\right]\right.}\right]^{2}\left(\mathrm{R}^{2}=0,982\right) . \\
& \mathrm{F}_{(\text {biótipo) }}=0,047^{\mathrm{ns} ;} \mathrm{F}_{\text {(biótipo x avaliação) }}=0,119^{\mathrm{ns}} .
\end{aligned}
$$


encerramento mais rápido do ciclo. Para o capim-colchão, a taxa de crescimento absoluto apresentou-se estável no início do ciclo, para ambos os biótipos, com rápido aumento a partir dos 30 DAE, em modelo de semi-parábola, com pico aos $80 \mathrm{DAE}$, aproximadamente, e sem diferenças significativas entre os biótipos para as diversas avaliações.

A ausência de diferenças na MS e na G sugere equivalência de crescimento entre biótipos e, portanto, equivalência competitiva. Assim, é provável que possuam a mesma competitividade, com a dominância numérica de um biótipo sobre o outro decorrente apenas da pressão de seleção causada pelo herbicida. Segundo Devine (1997), a adaptabilidade não é afetada pela mutação dos biótipos resistentes aos inibidores da ACCase e, assim, não apresentariam desvantagem de crescimento na ausência de pressão de seleção.

Na competição intra-específica dos biótipos $\mathrm{Re} \mathrm{S}$ (Figura 3) foi detectada baixa interação. Observa-se que as produções relativas, observadas para os biótipos $\mathrm{R} \mathrm{e} \mathrm{S,} \mathrm{e}$ a produção relativa total desviaram-se levemente da produção porcentual esperada $(20,40,60$ ou $80 \%)$. A variação da produção relativa do biótipo resistente foi menor, quando comparado ao biótipo suscetível. A maior queda de produção relativa, nas maiores proporções do biótipo $\mathrm{S}$, ocorreu em conseqüência da maior produção de massa de matéria seca do monocultivo desse biótipo. Neste caso, observa-se na Figura 3 que a habilidade de cada biótipo em interferir entre si é equivalente; isto é, cada biótipo contribui para a biomassa total produzida, em proporção equivalente à sua presença na mistura, o

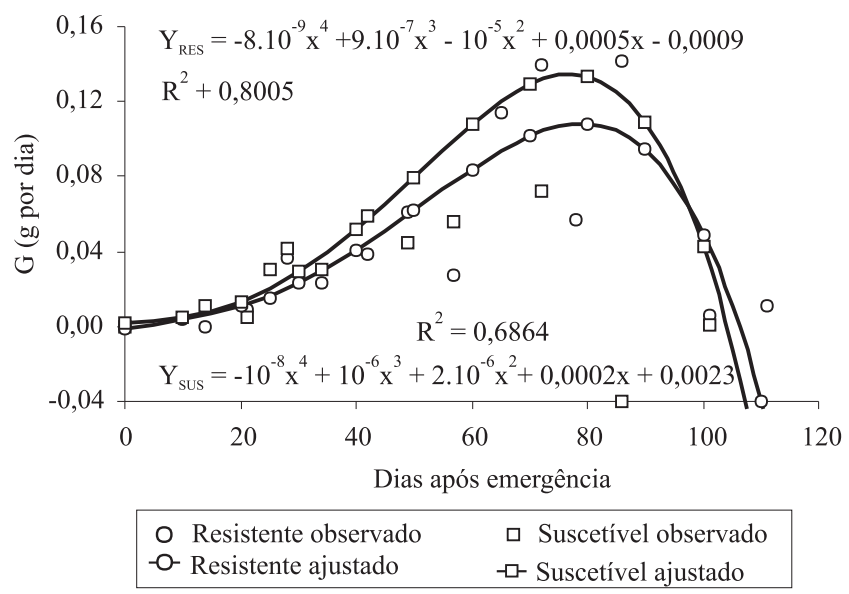

Figura 2. Taxa de crescimento absoluto $(\mathrm{G})$ dos biótipos de capim-colchão, resistente e suscetível aos herbicidas inibidores da ACCase, durante o ciclo de crescimento. A curva representa os valores ajustados da equação não linear, e os pontos a média dos valores observados. $\mathrm{F}_{\text {(biótipo) }}=0,012^{\text {ns. }}$; $\mathrm{F}_{\text {(biótipo } \mathrm{x} \text { avaliação) }}=0,107^{\mathrm{ns}}$. que está em concordância com o crescimento semelhante destes biótipos (Figuras 1 e 2).

Wiederholt \& Stoltenberg (1996) também conduziram experimentos com o objetivo de determinar a produtividade e a capacidade competitiva de um biótipo de capimcolchão (Digitaria sanguinalis), resistente aos inibidores da ACCase, quando comparado ao biótipo suscetível. Concluíram que a habilidade intra-específica e interespecífica dos biótipos resistentes e suscetíveis foi similar, pois a produtividade dos dois biótipos não diferiu, estatisticamente, em condições não competitivas e no campo.

Estudos atuais que avaliaram a competitividade intraespecífica de biótipos de plantas daninhas, resistentes e suscetíveis a herbicidas, são mais comuns quando relacionados aos inibidores da acetolactase sintase (ALS). Neste sentido, Christoffoleti (1992) desenvolveu pesquisa em casa de vegetação e em campo, em que não foram observadas diferenças na adaptabilidade ecológica de biótipos de Kochia scoparia resistentes e suscetíveis aos inibidores da ALS. Somente para a condição de casa de vegetação, Brighenti et al. (2001) e Christoffoleti (2001) chegaram às mesmas conclusões, com relação aos biótipos de Euphorbia heterophylla e Bidens pilosa, respectivamente.

A manutenção da habilidade competitiva pode estar relacionada com a mutação responsável pela resistência destes biótipos de plantas daninhas (insensibilidade

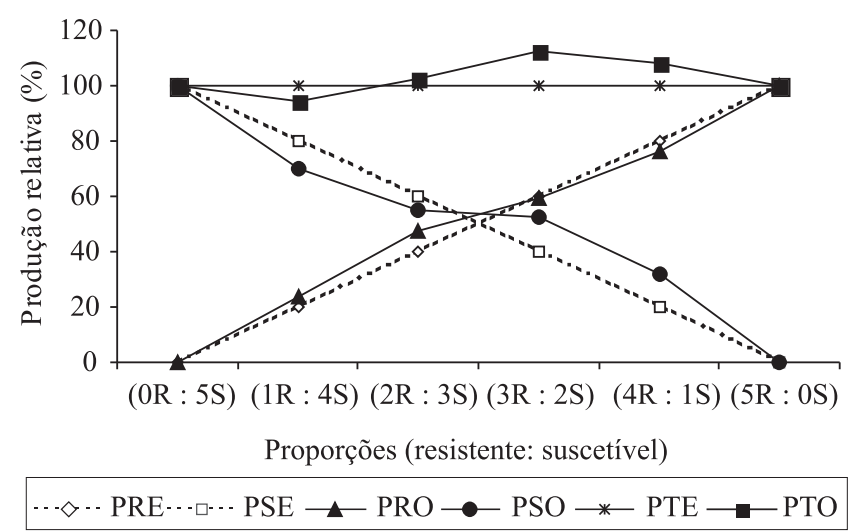

Figura 3. Rendimento relativo dos biótipos de capim-colchão, resistente (R) e suscetível (S) aos inibidores da ACCase, em função das diferentes proporções. PRE: produção relativa esperada do biótipo R; PSE: produção relativa esperada do biótipo S; PRO: produção relativa observada do biótipo R; PSO: produção relativa observada do biótipo $\mathrm{S}$; PTE: produção relativa esperada total; PTO: produção relativa observada total. 
enzimática), que não resulta em custo energético para o biótipo resistente. Em geral, as diferenças de adaptabilidade ecológica estão mais relacionadas com os casos de resistência aos herbicidas inibidores do fotossistema II (Christoffoleti \& López Ovejero, 2004). Quando a mutação que provoca a resistência está associada com a redução da adaptabilidade ecológica, a evolução da população resistente pode ser lenta (Jasieniuk \& Maxwell, 1994).

Em condição de campo, a diferenciação dos biótipos de plantas daninhas, resistentes e suscetíveis, não pode ser feita na ausência dos herbicidas. Portanto, em geral, compara-se a adaptabilidade ecológica de biótipos coletados em diferentes localidades, de modo que as conclusões obtidas são estimativas do que ocorre em campo. Segundo Christoffoleti \& López Ovejero (2004), a mesma adaptabilidade ecológica do biótipo resistente e suscetível indica que ocupam nichos ecológicos semelhantes e, dessa forma, uma vez estabelecida a população resistente, naturalmente ela não retorna para a frequiência original de suscetibilidade, o que tem sido observado para os casos de resistência aos inibidores da ALS e ACCase.

Observa-se que ocorreu competição interespecífica, quando a semeadura dos biótipos de capim-colchão e da soja foi realizada no mesmo dia (0 DAS), uma vez que os valores da produção obtidos na mistura das duas espécies se desviaram, significativamente, da linha de produção esperada (Figura 4). A soja produziu quantidade de matéria seca acima da esperada, enquanto a produção de matéria seca dos biótipos $\mathrm{R} \mathrm{e} \mathrm{S}$ mantevese abaixo do esperado. Assim, os gráficos revelam a existência de interação negativa o que indica que a competição entre as espécies ocorre pelos mesmos recursos e que a soja é mais agressiva na conquista destes recursos do que os biótipos de capim-colchão. Para os dois biótipos foi observado o mesmo comportamento. Em geral, a produção observada total foi levemente inferior à esperada, o que representa um antagonismo mútuo, com prejuízo para a produtividade máxima da maioria das proporções.

Quando a semeadura dos biótipos foi realizada 7 DAS, observou-se maior competição, uma vez que a produtividade total observada foi inferior à esperada, quando comparada a 0 DAS. Isto caracteriza, novamente, o antagonismo mútuo em que a produtividade máxima só foi obtida com as monoculturas (Figura 5). O comportamento da cultura da soja e dos biótipos $\mathrm{Re} S$ foram diferentes, quando comparadas as duas situações (0 DAS x 7 DAS).
A produção da soja observada foi praticamente a esperada, e a dos biótipos $\mathrm{R}$ e $\mathrm{S}$ foi menor que a esperada, no entanto, a redução em 7 DAS foi menos significativa, quando comparada a 0 DAS. Para os dois biótipos, foi observado o mesmo comportamento. Assim, o estabelecimento prévio dos biótipos $\mathrm{R}$ ou $\mathrm{S}$ de capim-colchão aumenta sua capacidade competitiva.

Christoffoleti \& Victoria Filho (1996), utilizando o método substitutivo, observaram que o milho (Zea mays L.) foi um competidor muito mais agressivo que o caruru (Amaranthus retroflexus), e para a planta cultivada, a competição intra-específica foi mais importante que a competição interespecífica. Rizzardi et al. (2004), ao utilizar
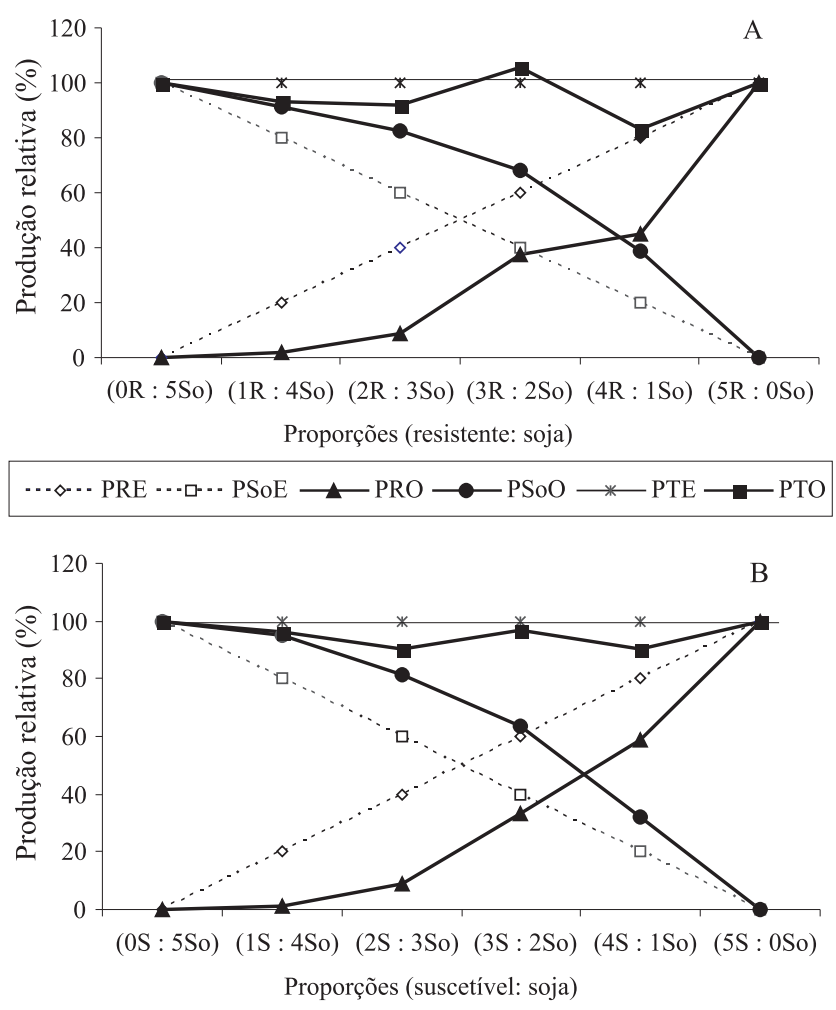

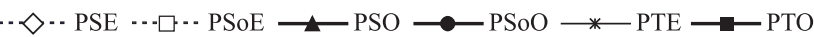

Figura 4. Rendimento relativo dos biótipos resistente (A) e suscetível (B) de capim-colchão e da cultura da soja, em função das diferentes proporções, quando semeados no mesmo dia (0 DAS). PRE: produção relativa esperada do biótipo R; PSoE: produção relativa esperada da cultura da soja; PRO: produção relativa observada do biótipo R; PSoO: produção relativa observada da cultura da soja; PTE: produção relativa esperada total; PTO: produção relativa observada total; PSE: produção relativa esperada do biótipo S; PSO: produção relativa observada do biótipo S. 
o método substitutivo, verificaram que a redução na massa da soja foi mais intensa, quando em presença da corda-deviola (Ipomoea ramosissima) do que de amendoim-bravo (Euphorbia heterophylla) e, principalmente, quando as plantas daninhas se estabeleceram antes da cultura.

Rizzardi (2002), ao estudar as perdas de rendimento causadas pelo atraso na semeadura da soja, em relação à dessecação da cobertura vegetal, observou que as perdas de produtividade, causadas pela presença de 50 plantas $\mathrm{m}^{-2}$ de picão-preto (Bidens sp.) ou guanxuma (Sida rhombifolia L.), foram menos intensas quanto mais próxima foi a dessecação da cobertura vegetal em relação à semeadura da soja. Esses estudos reforçam a

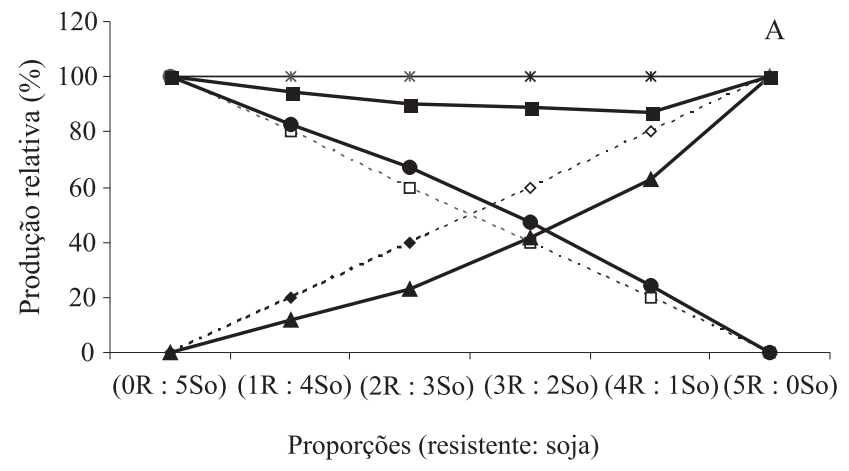

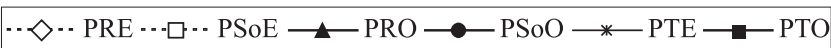

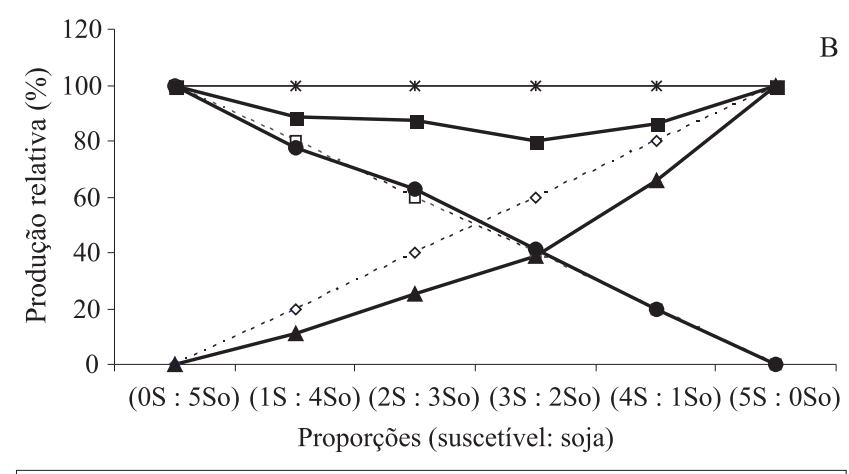

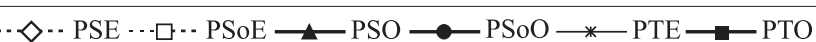

Figura 5. Rendimento relativo dos biótipos resistente (A) e suscetível (B) de capim-colchão e da cultura da soja semeada sete dias após (7 DAS), em função das diferentes proporções. PRE: produção relativa esperada do biótipo R; PSoE: produção relativa esperada da cultura da soja; PRO: produção relativa observada do biótipo R; PSoO: produção relativa observada da cultura da soja; PTE: produção relativa esperada total; PTO: produção relativa observada total; PSE: produção relativa esperada do biótipo S; PSO: produção relativa observada do biótipo $\mathrm{S}$. importância de se realizar a dessecação no momento adequado. No entanto, é importante lembrar que existem grandes diferenças na habilidade competitiva de cultivares de soja.

\section{Conclusão}

Os biótipos de capim-colchão, resistente e suscetível aos herbicidas inibidores da acetil coenzima A carboxilase, apresentam crescimento e competitividade semelhantes, o que sugere que ambos os biótipos possuem a mesma adaptabilidade ecológica.

\section{Referências}

AGUILERA, D.B.; FERREIRA, F.A.; CECON, P.R. Crescimento de Siegesbeckia orientalis sob diferentes condições de luminosidade. Planta Daninha, v.22, p.43-51, 2004.

BRIGHENTI, A.M.; GAZZIERO, D.L.P.; VOLL, E.; ADEGAS, F.S.; VAL, W.M.C. Análise de crescimento de biótipos de amendoimbravo (Euphorbia heterophylla) resistente e suscetível aos herbicidas inibidores da ALS. Planta Daninha, v.19, p.51-59, 2001.

CALBO, A.G.; SILVA, W.L.C.; TORRES, A.C. Comparação de modelos e estratégias para análise de crescimento. Revista Brasileira de Fisiologia Vegetal, v.1, p.1-7, 1989.

CANTO-DOROW, T.S. O gênero Digitaria Haller (Poaceae Panicoideae - Poniceae) no Brasil. 2001. 386p. Tese (Doutorado) - Universidade Federal do Rio Grande do Sul, Porto Alegre.

CHRISTOFFOLETI, P.J. Análise comparativa do crescimento de biótipos de picão-preto (Bidens pilosa) resistente e suscetível aos herbicidas inibidores da ALS. Planta Daninha, v.19, p.75-83, 2001.

CHRISTOFFOLETI, P.J. Growth, competitive ability, and fitness of sulfonylurea resistant and susceptible Kochia scoparia biotypes. 1992. 198p. Tese (Doutorado) - Colorado State University, Fort Collins.

CHRISTOFFOLETI, P.J.; LÓPEZ OVEJERO, R.F. Definições e situação da resistência de plantas daninhas a herbicidas no Brasil e no mundo. In: CHRISTOFFOLETI, P.J. (Coord.). Aspectos de resistência de plantas daninhas a herbicidas. 2.ed. Campinas: HRAC-BR, 2004. p.3-22.

CHRISTOFFOLETI, P.J.; VICTORIA FILHO, R. Efeitos da densidade e proporção de plantas de milho (Zea mays L.) e caruru (Amaranthus retroflexus L.) em competição. Planta Daninha, v.14, p.42-47, 1996.

CHRISTOFFOLETI, P.J.; WESTRA, P.; MOORE III, F. Growth analyses of sulfonylurea-resistant and susceptible kochia (Kochia scoparia). Weed Science, v.45, p.691-695, 1997.

COUSENS, R. Aspects of the design and interpretation of competition (interference) experiments. Weed Technology, v.5, p.664-673, 1991.

DEVINE, M.D. Mechanisms of resistance to acetyl-coenzime A carboxylase inhibitors: a review. Pesticide Science, v.51, p.259264, 1997. 
FLECK, N.G.; RIZZARDI, M.A.; VIDAL, R.A.; MEROTTO JÚNIOR, A.; AGOSTINETTO, D.; BALBINOT JUNIOR, A.A. Período crítico para controle de Brachiaria plantaginea em função de épocas de semeadura da soja após dessecação da cobertura vegetal. Planta Daninha, v.20, p.53-62, 2002.

HARPER, J.L. Mixtures of species. I. Space and proportions. In: HARPER, J.L. (Ed.). Population biology of plants. $8^{\text {th }}$ ed. London: Academic Press, 1977. p.237-276.

HOLT, J.S.; RADOSEVICH, S.R. Differential growth of two common groundsel (Senecio vulgaris) biotypes. Weed Science, v.31, p.112-120, 1983.

JASIENIUK, M.; BRÛLÉ-BABEL, A.L.; MORRISON, I.N. The evolution and genetics of herbicide resistance in weeds. Weed Science, v.44, p.176-193, 1996.

JASIENIUK, M.; MAXWELL, B.D. Population genetics and the evolution of herbicide resistance in weeds. Phytoprotection, v.75, p.25-35, 1994.

LÓPEZ OVEJERO, R.F.; CARVALHO, S.J.P.; PENCKOWSKI, L.H.; NICOLAI, M.; CHRISTOFFOLETI, P.J. Resistência de populações de capim-colchão (Digitaria ciliaris) aos herbicidas inibidores da acetil CoA carboxilase. Planta Daninha, v.23, p.543549, 2005.

LÓPEZ OVEJERO, R.F.; CHRISTOFFOLETI, P.J.; VARGAS, L. Resistência de plantas daninhas a herbicidas. In: VARGAS, L.; ROMAN, E.S. (Ed.). Manual de manejo e controle de plantas daninhas. Bento Gonçalves: Embrapa Uva e Vinho, 2004. p.185214.

RADOSEVICH, S.R. Methods to study interactions among crops and weeds. Weed Technology, v.1, p.190-198, 1987.
RADOSEVICH, S.R.; ROUSH, M.L. The role of competition in agriculture. In: GRACE, J.B.; TILLMANN, D. (Ed.). Perspectives on plant competition. San Diego: Academic Press, 1990. p.341-363.

RIZZARDI, M.A. Nível de dano econômico para tomada de decisão no controle de picão-preto (Bidens spp.) e guanxuma (Sida rhombifolia L.) na cultura de soja. 2002. 175p. Tese (Doutorado) - Universidade Federal do Rio Grande do Sul, Porto Alegre.

RIZZARDI, M.A.; ROMAN, E.S.; BOROWSKI, D.Z.; MARCON, R. Interfererência de populações de Euphorbia heterophylla e Ipomoea ramosissima isoladas ou em misturas sobre a cultura de soja. Planta Daninha, v.22, p.29-34, 2004.

SPADOTTO, C.A.; MARCONDES, D.A.S.; LUIZ, A.J.B.; SILVA, C.A.R. Determinação do período crítico para prevenção da interferência de plantas daninhas na cultura da soja: uso do modelo "broken-stick". Planta Daninha, v.12, p.59-62, 1994.

VIDAL, R.A.; TREZZI, M.M. Análise de crescimento de biótipos de leitera (Euphorbia heterophylla) resistentes e suscetível aos herbicidas inibidores da ALS. Planta Daninha, v.18, p.427-433, 2000.

WARWICK, S.I.; BLACK, L.D. Relative fitness of herbicideresistant and susceptible biotypes of weeds. Phytoprotection, v.75, p.37-49, 1994.

WIEDERHOLT, R.J.; STOLTENBERG, D.E. Similar fitness between large crabgrass (Digitaria sanguinalis) accessions resistant or susceptible to acetyl-coenzyme A carboxylase inhibitors. Weed Technology, v.10, p.42-49, 1996.

WIT, C.T. de; VAN DEN BERG, J.P. Competition between herbage plants. Netherlands Journal of Agricultural Science, v.13, p.212221, 1965. 